\section{Radiotherapie nach Lumpektomie bei Seniorinnen häufig überflüssig}

Kann man bei älteren Frauen mit kleineren Östrogenrezeptor-(ER) positiven Mammakarzinomen nach Lumpektomie und unter antihormoneller Therapie auf die adjuvante Radiotherapie verzichten? Langzeitergebnisse einer randomisierten Studie unterstützen ein solches Vorgehen, was das Gesamtüberleben betrifft, weil lediglich beim Lokoregionären Rezidivrisiko ein Vorteil der Strahlentherapie beobachtet werden konnte.

$\mathrm{D}$ ie von der Cancer and Leukemia Group B (CALGB) initiierte randomisierte Studie CALGB 9343 wurde zwischen Juli 1994 und Februar 1999 durchgeführt. Darin einbezogen waren 636 Frauen ( $\geq 70$ Jahre) mit einem ERpositiven Mammakarzinom im klinischen Stadium I (T1N0M0 entsprechend der TNM-Klassifikation), die nach Brust erhaltender Chirurgie randomisiert entweder Tamoxifen plus Strahlentherapie (TamRT, $\mathrm{n}=317$ ) oder nur Tamoxifen (Tam, $\mathrm{n}=319$ ) erhalten hatten.

Die mediane Nachbeobachtungszeit beträgt jetzt 12,6 Jahre. Nach zehn Jah- ren waren $98 \%$ (95\%-Konfidenzintervall [95\%-KI] 96-99\%) der Frauen aus der TamRT-Gruppe und $90 \%$ (95\%-KI 85-93\%) der Frauen aus der Tam-Gruppe frei von lokoregionären Rezidiven geblieben. Dieser Unterscheid von $8 \%$ ist statistisch signifikant (Hazard Ratio [HR] 0,18, $95 \%$-KI 0,07-0,42; p < 0,001). Bei den weiteren primären Endpunkten Zeit bis zur Mastektomie, Zeit bis zum Auftreten von Metastasen, brustkrebsspezifisches Überleben und Gesamtüberleben bestanden keine signifikanten Unterschiede zwischen den Gruppen. Das 10-Jahres-Gesamtüberleben lag bei $67 \%$ (95\%-KI 62-72\%) in der TamRT-
Gruppe und bei $66 \%$ (95\%-KI 61-71\%) in der Tam-Gruppe.

Insgesamt wurde mit diesem Langzeit-Follow-up die Ergebnisse des 5-Jahres-Follow-ups bestätigt. Auch dort zeigte sich ein kleiner Vorteil für die adjuvante Strahlentherapie in der Verhinderung von lokoregionären Rezidiven (und von ipsilateralen Rezidiven). Bei den übrigen Zielvariablen bestanden keine Unterschiede.

Fazit: Bei Frauen im Alter von über 70 Jahren mit einem ER-positiven Mammakarzinom im Stadium I hat eine Strahlentherapie nach Lumpektomie plus adjuvanter antihormoneller Therapie keine Vorteile bei Gesamtüberleben, Auftreten von Metastasen, krankheitsfreiem Überleben oder Brusterhaltung. Eine Strahlentherapie kann lediglich die Rate von lokoregionären Rezidiven etwas verringern.

Barbara Kreutzkamp

Hughes KS et al. Lumpectomy plus tamoxifen with or without irradiation in women age 70 years or older with early breast cancer: Long term follow-up of CALGB 9343. J Clin Oncol. 2013 May 20. [Epub ahead of print].

\title{
Trastuzumab: Auch Frauen mit ILC profitieren
}

Ob Frauen mit HER2-positivem invasivem lobulärem Mammakarzinom (ILC) genauso wie HER2-positive Frauen mit duktalen Karzinomen von Anti-HER2Therapien profitieren, ist unklar. Eine Studie untersuchte dies am Beispiel von Trastuzumab.

Eür den Outcome-Vergleich einer - Trastuzumab-Therapie bei HER2-positiven lobulären und duktalen Mammakarzinomen (IDC) wurde auf Daten der Herceptin-Adjuvant-(HERA) Studie zurückgegriffen. Darin wurden Patientinnen mit HER2-positivem Mammakarzinom nach Chirurgie, ggf. Radiotherapie und Chemotherapie randomisiert entweder einer Beobachtung oder in einen der Trastuzumab-Studienarme (ein oder zwei Jahre Trastuzumab) zugewiesen. In die vorliegende retrospektive Auswertung wurden 3.401 Frauen mit ILC oder IDC einbezogen, die entweder beobachtet worden waren oder ein Jahr Trastuzumab erhalten hatten. Die Lokalisation des ersten Rezidivs (z. B. lokal oder regi- onal, kontralateral) wurde ermittelt und mithilfe von Cox-proportional-hazardModellen das krankheitsfreie Überleben (DFS) und das Gesamtüberleben (OS) errechnet.

Die mediane Nachbeobachtungszeit betrug vier Jahre. Insgesamt wurden die Daten von 187 ILC-Patientinnen und 3.213 IDC-Patientinnen ausgewertet. Hohe Allred-Scores (6-8) waren bei Frauen mit ILC häufiger als bei Frauen mit IDC und zwar sowohl für die Östrogenrezeptoren (36,9 vs. $22,7 \%$ ) als auch die Progesteronrezeptoren (44,1 vs. $28,5 \%)$. In der ILC-Gruppe bestand ein Trend in Richtung abnehmender Zahl von HER2-Kopien. Die Lokalisationsmuster des ersten Rezidivs waren in der
ILC- und IDC-Gruppe vergleichbar. Die Hazard Ratios (HR) für den Vergleich ein Jahr Trastuzumab versus Beobachtung betrug bei den ILC-Patientinnen 0,63 (95\%-Konfidenzintervall [95\%-KI] $0,34-1,15)$ und für die IDC-Patientinnen 0,77 (95\%-KI 0,67-0,89; p für Interaktion $=0,49)$. Die HR für das Gesamtüberleben im Vergleich ein Jahr Trastuzumab vs. Beobachtung lag beim ILC bei 0,60 (95\%-KI 0,27-1,31) und beim IDC bei 0,86 (95\%-KI 0,71-1,06; p für Interaktion $=0,29)$.

Fazit: Frauen mit einem HER2-positiven ILC profitieren genauso wie Frauen mit HER2-positivem IDC von einer adjuvanten Trastuzumab-Therapie. Das ist das Ergebnis einer retrospektiven Auswertung der HERA-Studie.

Barbara Kreutzkamp

Metzger-Filho $\mathrm{O}$ et al. Magnitude of trastuzumab benefit in patients with HER2-positive, invasive lobular breast carcinoma: Results from the HERA trial. J Clin Oncol. 2013;31(16):1954-60. 